\title{
Caracterização dos referenciais das dissertações e teses dos programas mineiros de pós- graduação
}

\author{
Characterization of references in dissertations and theses from the graduate programs in Minas \\ Gerais
}

\section{Caracterización de las referencias de las disertaciones y tesis de los programas de posgrado de Minas Gerais}

\section{RESUMO}

Objetivo: Caracterizar os referenciais teórico/metodológico das dissertações/teses com abordagem qualitativa e mista, dos Programas de Pós-graduação Stricto sensu em Enfermagem públicos mineiros. Método: Descritivo, retrospectivo e documental, com acesso às bibliotecas digitais dos Programas. Identificou-se 888 dissertações e 164 teses no período de 1996 a 2019. Dados coletados por instrumento autoral e analisados pela estatística descritiva. Resultados: Das 888 dissertações, 503 (56,64\%) delineamento quantitativo, 345 (38,86\%) qualitativo e 40 (4,5\%) misto/quali-quanti. Das 164 teses, 92(56,10\%) delineamento quantitativo, 64 (39,02\%) qualitativo e 8 (4,88\%) misto/quali-quanti. Referenciais predominantes: Análise temática e de Conteúdo/Bardin/Minayo; Fenomenologia; Estudo de Caso; Teoria das Representações Sociais/Análise Crítica do Discurso; Hermenêutica-Dialética; Análise Compreensiva; Sociologia Compreensiva do Cotidiano, Etnografia, Análise Estrutural da Narração e Teoria Fundamentada nos Dados. Conclusão: Esses referenciais contribuem para translação do conhecimento e qualidade do cuidado de Enfermagem.

Descritores: Enfermagem; Pesquisa em Enfermagem; Características dos Estudos; Programas de Pós-graduação em Saúde; Educação de Pós-graduação em Enfermagem.

\section{ABSTRACT}

Objective: To characterize the theoretical/methodological references of theses/ dissertations with qualitative and mixed approach of Postgraduate Programs in Nursing at Federal Universities in Minas Gerais. Method: Descriptive, retrospective and documentary study, carried out by accessing digital libraries of the programs. The sample consisted of 888 dissertations and 164 theses between 1996 and 2019. Data collected by authorial instrument and analyzed by descriptive statistics. Results: Out of the 888 dissertations, 503 (56.64\%) quantitative design, 345 (38.86\%) qualitative, and 40 (4.5\%) mixed/quali-quanti. Out of the 164 theses, 92 (56.10\%) quantitative design, 64 (39.02\%) qualitative and 8 (4.88\%) mixed/quali-quanti. Predominant references: Thematic and Content Analysis/Bardin/Minayo; Phenomenology; Case study; Theory of Social Representations/Critical Discourse Analysis; Hermeneutics-Dialectic; Comprehensive Analysis; Comprehensive Sociology of Everyday Life, Ethnography, Structural Analysis of Narration and Grounded Theory. Conclusion: These references contribute to the translation of knowledge and quality of nursing care.

Descriptors: Nursing; Nursing Research; Study Characteristics; Health Graduate Programs; Education, Nursing, Graduate.

\section{RESUMEN}

Objetivo: Caracterizar los referentes teóricos/metodológicos de las disertaciones/tesis con enfoque cualitativo y mixto, de programas de posgrado Stricto sensu en enfermería, de universidades públicas de Minas Gerais. Método: Descriptivo, retrospectivo y documental, con acceso a las bibliotecas digitales de los programas. Fueron identificadas 888 disertaciones y 164 tesis, desde 1996 hasta 2019. Datos recogidos mediante instrumento autoral y analizados por estadística descriptiva. Resultados: De las 888 disertaciones, 503 $(56,64 \%)$ presentaron diseño cuantitativo; 345 (38,86\%) cualitativo y $40(4,5 \%)$ mixto. De las 164 tesis, $92(56,10 \%)$ con diseño cuantitativo; 64 (39,02\%) cualitativo y 8 (4,88\%) mixto. Referencias predominantes: Análisis Temático y de Contenido/Bardin/Minayo; Fenomenología; Estudio de caso; Teoría de las Representaciones Sociales/Análisis Crítico del Discurso; Hermenéutica-Dialéctica; Análisis comprensivo; Sociología Comprensiva de lo Cotidiano, Etnografía, Análisis Estructural de la Narración y Teoría Fundamentada en Datos. Conclusión: Estos referentes contribuyen a la traslación del conocimiento y calidad del cuidado de enfermería.

Descriptores: Enfermería; Investigación en Enfermería; Características del Estudio; Programas de Posgrado en Salud; Educación de Posgrado en Enfermería.

Munyra Rocha Silva Assunção

0000-0002-0998-3125

Bianca de Moura PelosoCarvalho ${ }^{1}$

0000-0001-5336-2249 Nathália Gianini Nery ${ }^{1}$ 0000-0002-6148-0613

Bianca Aparecida Brito da Silva ${ }^{1}$

0000-0001-8954-7616

\section{Eliza Maria Rezende Dázio ${ }^{1}$} 0000-0001-9216-6283

\section{Silvana Maria Coelho Leite \\ Fava $^{1}$ \\ 0000-0003-3186-9596}

${ }^{1}$ Universidade Federal de Alfenas, MG, Brasil.

Autor correspondente: Munyra Rocha Silva Assunção E-mail: munyrarsilva@hotmail.com

\section{Como citar este artigo:}

Assunção MRS, Peloso-Carvalho BM, Nery NG, et al. Caracterização dos referenciais das dissertações e teses dos programas mineiros de pós-graduação. Revista de Enfermagem do Centro-Oeste Mineiro. 2021;10:e3984. [Access___]; Available in: DOI: http://doi.org/10.19175/recom.v1 $\underline{0 i 0.3984}$ 


\section{INTRODUÇÃO}

A enfermagem é um campo de conhecimento científico, tecnológico e de inovação e uma prática social comprometida com as políticas públicas de atenção à saúde. Como ciência, ela faz conexões com diversos campos do saber, visando às potencialidades para a (re) construção de conhecimentos para atender as diversidades que permeiam o saber e as práticas da profissão(1).

Nesse contexto, a produção do conhecimento da área de Enfermagem tem sido realizada, principalmente pelos Programas de Pósgraduação Stricto sensu, o que tem contribuído para práticas inovadoras, contextualizadas e congruentes às políticas públicas, às necessidades das pessoas e para a visibilidade da área no cenário nacional e internacional ${ }^{(2)}$.

A construção do conhecimento tem se pautado nas abordagens quantitativa, qualitativa, quanti-qualitativa e mista e nos referenciais teórico e metodológico das ciências da saúde, humanas e sociais, dentre outras, para a investigação de seus objetos de estudo, assim como, para promover a translação do conhecimento, a divulgação e a utilização de resultados de pesquisa ${ }^{(3)}$. Optou-se neste estudo pela caracterização dos referenciais teórico/ metodológico das pesquisas com abordagem qualitativa e mista.

A pesquisa com abordagem qualitativa inicia com pressupostos e a utilização de estruturas teóricas/interpretativas/reflexivas que apresentam o estudo dos problemas da pesquisa, com o propósito de apreender os significados que a pessoa atribui a um problema social ou humano, bem como, compreender múltiplas visões subjetivas. Para a construção do conhecimento, ela demanda um raciocínio complexo, permeado entre o indutivo e dedutivo, e o pesquisador é tido como instrumento-chave, mediante o ambiente/contexto social, histórico e político dos participantes da pesquisa. Exige-se portanto do pesquisador a sustentação teórica, o rigor metodológico e a sua criatividade ${ }^{(4)}$.

O desenho de método misto/Quali-Quanti tem alcançado a sua visibilidade, nos últimos anos, uma vez que permite a complementariedade da pesquisa qualitativa para a pesquisa quantitativa e vice-versa. A associação de dados amplia o foco de discussão do estudo, propiciando a compreensão do todo, com suas inúmeras facetas existentes, apresentando maior clareza de resultados ${ }^{(5)}$.

O interesse em desenvolver este estudo parte da nossa aproximação com os referenciais metodológicos apreendidos na disciplina de Fundamentos Metodológicos do Processo de Cuidar e da Pesquisa de um Programa de Pósgraduação Stricto sensu em Enfermagem, para compreender os direcionamentos da pesquisa em Enfermagem e suas interfaces com outros saberes.

Optou-se pelo recorte da produção científica mineira, tendo em vista a parceria entre os Programas de Pós-graduação Stricto sensu em Enfermagem das universidades federais. Nesse contexto, buscou-se a produção dos Programas de Pós-graduação Stricto Sensu da Universidade Federal de Minas Gerais (UFMG), da Universidade Federal do Triângulo Mineiro (UFTM), de Juiz de Fora (UFJF), da Universidade Federal de São João Del Rei (UFSJ) e da Universidade Federal de Alfenas (UNIFAL-MG), com o objetivo de caracterizar os referenciais teórico/metodológico adotados nas teses e nas dissertações com abordagens qualitativa e mista dos Programas de Pós-graduação Stricto sensu em Enfermagem das Universidades Federais de Minas Gerais.

\section{MÉTODO}

Trata-se de estudo documental, descritivo e retrospectivo. O estudo documental consiste em identificar, verificar e apreciar os documentos, com vista a obter dados pertinentes. Extrai-se um reflexo objetivo da fonte original, possibilitando a localização, identificação, organização e avaliação das informações incluídas no documento, além da contextualização dos fatos em determinados momentos ${ }^{(6)}$.

A amostra foi constituída pelas teses e dissertações disponíveis no banco de teses e dissertações das universidades, sem recorte temporal.

Para a coleta de dados foi elaborado um instrumento com os seguintes dados: número de defesas de dissertações e teses, ano de defesa, programa, área de concentração, linhas de pesquisa, referenciais teórico/metodológico. Os dados foram coletados nos meses de novembro e dezembro de 2019. Procedeu-se inicialmente a leitura do resumo e, quando necessário, da dissertação e da tese na íntegra, na busca por informações, com vista a responder os objetivos do estudo. Os dados foram organizados em uma 
planilha construída no Programa Microsoft Excel 2016 e analisados por meio de estatística descritiva.

\section{RESULTADOS E DISCUSSÃO}

Foram levantadas um total de 1239 teses e dissertações dos Programas de Pós-graduação Stricto sensu em Enfermagem das Universidades Federais de Minas Gerais, das quais, 1064 dissertações e 175 teses, distribuídas nos seguintes programas: 176 dissertações e 23 teses (UFTM); 128 dissertações (UFJF); 620 dissertações e 152 teses (UFMG); 56 dissertações (UFSJ) e 84 dissertações (UNIFAL-MG). Contudo, 176 dissertações $(16,54 \%)$ e 11 teses (6,29\%) não estavam disponíveis nas páginas dos Programas. Desse modo, fizeram parte deste estudo 888 dissertações e 164 teses, totalizando uma amostra de 1052, conforme apresentado na Tabela 1.

Tabela 1 - Distribuição das dissertações e teses dos Programas de Pós-graduação Stricto sensu em Enfermagem das Universidades Federais de Minas Gerais, Alfenas, 2019

\begin{tabular}{lcccccccc}
\hline Variáveis & $\begin{array}{c}\text { Número } \\
\text { de Defesas }\end{array}$ & $\begin{array}{c}\text { Indisponíveis na } \\
\text { página do } \\
\text { Programa }\end{array}$ & Analisadas & UFTM & UFJF & UFMG & UFSJ & UNIFAL \\
\hline Dissertações & 1064 & 176 & 888 & 176 & 128 & 620 & 56 & 84 \\
Teses & 175 & 11 & 164 & 23 & - & 152 & - & - \\
Total & 1239 & 187 & 1052 & 199 & 128 & 772 & 56 & 84 \\
\hline
\end{tabular}

Fonte: das autoras.

Constatou-se que, no período de 1996 a 2019, foram defendidas 1064 dissertações e, no período de 2008 a 2019, 175 teses.

Com relação à abordagem metodológica, verificou-se o predomínio do delineamento quantitativo tanto para as dissertações como para as teses, e a escassez de estudo com método misto/Quali-Quanti, conforme Tabela 2.

Tabela 2 - Distribuição das dissertações e teses segundo a abordagem metodológica dos Programas de Pós-graduação Stricto sensu em Enfermagem das Universidades Federais de Minas Gerais, Alfenas, 2019

\begin{tabular}{lcccc}
\hline \multirow{2}{*}{ Abordagem Metodológica } & \multicolumn{2}{c}{ Dissertações } & \multicolumn{2}{c}{ Teses } \\
\cline { 2 - 5 } & $\mathrm{N}$ & 56,64 & $\mathrm{n}$ & $\%$ \\
\hline Quantitativa & 503 & 38,86 & 64 & 56,10 \\
Qualitativa & 345 & 4,5 & 8 & 39,02 \\
Mista/Quali-Quanti & 40 & 4,88 & \\
\hline
\end{tabular}

Fonte: das autoras.

Em relação aos referenciais teórico/metodológico adotados nas dissertações e teses com abordagem qualitativa e mista, constatou-se a utilização de 125 referenciais. Os referenciais mais utilizados nas dissertações e teses, de acordo com os Programas, estão apresentados no Quadro 1.

Quadro 1 - Distribuição dos Referenciais teórico-metodológico mais utilizados nas dissertações e teses dos Programas de Pósgraduação Stricto sensu em Enfermagem das Universidades Federais de Minas Gerais, Alfenas, 2019

\begin{tabular}{|c|c|c|c|c|c|c|}
\hline Referenciais Teórico-Metodológicos & $\begin{array}{c}\text { Número total } \\
\text { (teórico e } \\
\text { metodológico) }\end{array}$ & UFMG & UFTM & UFJF & UFS & UNIFAL \\
\hline Estudo de Caso & 56 & $\mathrm{X}$ & $\mathrm{X}$ & $\mathrm{X}$ & $\mathrm{X}$ & $\mathrm{X}$ \\
\hline $\begin{array}{c}\text { Análise temática ou de Conteúdo } \\
\text { Bardin ou Minayo }\end{array}$ & 210 & $\mathrm{X}$ & $\mathrm{X}$ & $\mathrm{X}$ & $\mathrm{X}$ & $\mathrm{X}$ \\
\hline Teoria das Representações Sociais & 54 & $\mathrm{X}$ & $\mathrm{X}$ & $\mathrm{X}$ & & $\mathrm{X}$ \\
\hline Análise Crítica do Discurso & 20 & $\mathrm{X}$ & & $\mathrm{X}$ & & \\
\hline
\end{tabular}


4|Assunção MRS, Peloso-Carvalho BM, Nery NG, et al.

Quadro 1 - Distribuição dos Referenciais teórico-metodológico mais utilizados nas dissertações e teses dos Programas de Pósgraduação Stricto sensu em Enfermagem das Universidades Federais de Minas Gerais, Alfenas, 2019

\begin{tabular}{|c|c|c|c|c|c|c|}
\hline Referenciais Teórico-Metodológicos & $\begin{array}{l}\text { Número total } \\
\text { (teórico e } \\
\text { metodológico) }\end{array}$ & UFMG & UFTM & UFJF & UFSJ & UNIFAL \\
\hline $\begin{array}{l}\text { Fenomenologia ou Fenomenologia de } \\
\text { Martin Heidegger }\end{array}$ & 68 & $x$ & $x$ & $x$ & $x$ & $x$ \\
\hline Etnografia & 24 & $x$ & $x$ & $x$ & & $x$ \\
\hline $\begin{array}{c}\text { Hermenêutica-Dialética } \\
\text { ou Dialética }\end{array}$ & 33 & $x$ & $x$ & $x$ & & $x$ \\
\hline Análise Compreensiva & 28 & $\mathrm{X}$ & & $x$ & & \\
\hline Teoria Fundamentada nos Dados & 18 & $\mathrm{X}$ & & $x$ & $x$ & $x$ \\
\hline Análise Estrutural da Narração & 17 & $x$ & & $x$ & & \\
\hline $\begin{array}{c}\text { Sociologia Compreensiva do } \\
\text { Cotidiano e/ou Sociologia } \\
\text { Compreensiva do Cotidiano de } \\
\text { Maffesoli }\end{array}$ & 33 & $x$ & & $x$ & $x$ & \\
\hline
\end{tabular}

Fonte: Das autoras.

Outras referenciais teórico/metodológico também merecem destaque, como a Educação crítica e libertadora de Paulo Freire, Histórico Cultural de Vigotski, Pesquisa Convergente Assistencial (PCA) e as Teorias de Enfermagem, principalmente a Teoria do Autocuidado de Dorothea Orem e a Teoria da Diversidade Cultural de Madeleine Leininger.

Os dados apontam que os pesquisadores, para a produção do conhecimento da Área de Enfermagem, têm buscado por uma integração interdisciplinar com os aportes das Ciências Sociais, da Antropologia e da Psicologia, o que tem contribuído para alcançar um olhar mais complexo sobre o ser humano e os fenômenos do adoecimento, da vida e da morte.

Em conformidade com os dados da Coordenação de Aperfeiçoamento de Pessoal de Nível Superior (CAPES), atualmente são oferecidos 79 Programas de Pós-graduação Stricto sensu em Enfermagem, e os Programas das Universidades Federais de Minas Gerais representam 6,3\% desses programas com a oferta de oito cursos.

O Programa de Pós-graduação Stricto sensu em Enfermagem da Escola de Enfermagem da Universidade Federal de Minas (UFMG) oferece os cursos de Mestrado e de Doutorado acadêmicos. O curso de Mestrado tem a área de concentração "Saúde e Enfermagem", com três linhas de pesquisa: "Cuidar em Saúde e Enfermagem", "Gestão e Educação na Saúde e Enfermagem" e "Epidemiologia, Políticas e Práticas em Saúde das Populações". O curso de Doutorado em Enfermagem oferece três linhas de pesquisa: "Cuidar em Saúde e Enfermagem", "Gestão e Educação na Saúde e Enfermagem" e
"Epidemiologia, Políticas e Práticas de Saúde das Populações".

O Programa de Pós-graduação Stricto sensu em Atenção à Saúde da Universidade Federal do Triângulo Mineiro (UFTM) oferece os cursos de Mestrado e Doutorado acadêmicos, com área de concentração em "Saúde e Enfermagem" e as linhas de pesquisa "Atenção à Saúde das Populações" e "O Trabalho na Saúde e na Enfermagem"

O Programa de Pós-graduação Stricto sensu Universidade Federal de Juiz de Fora (UFJF) oferece o curso de Mestrado em Enfermagem com a área de concentração "Cuidado em Saúde e Enfermagem" com as linhas de pesquisa: "Fundamentos Teóricos, Políticos e Culturais do Cuidado em Saúde e Enfermagem" e "Tecnologia e Comunicação no Cuidado em Saúde e Enfermagem".

O Programa de Pós-graduação Stricto sensu Mestrado e Doutorado em Enfermagem da Universidade Federal de Alfenas (UNIFAL-MG), com uma área de concentração, "Enfermagem", oferece duas linhas de pesquisa: "Processo de cuidar em Enfermagem" e "Gestão em serviços de Saúde e Educação". Cabe destacar que o curso de doutorado iniciou em dezembro de 2020.

O Programa de Pós-graduação Mestrado Acadêmico em Enfermagem da Universidade Federal de São João Del-Rei (UFSJ), com área de concentração, "Enfermagem", oferece duas linhas de pesquisa: "Gestão, Organização e Avaliação em Saúde e Enfermagem" e "O Processo de Cuidar em Saúde e Enfermagem".

As dissertações e teses constituem um requisito parcial para a concessão do título de mestre e doutor. Os egressos desses programas 
devem ser capazes de produzir e disseminar conhecimentos que elevam a qualidade de vida da sociedade; de diversificar os ideais, efetivar o exercício da cidadania para a qualificação do cuidado e para as reflexões da essência da Enfermagem ${ }^{(7)}$.

Quanto à abordagem, verificou-se o predomínio dos estudos quantitativos, resultado similar ao encontrado em estudo que teve por objetivo analisar a produção do conhecimento do Programa de Pós-graduação em Enfermagem da Universidade Estadual de Campinas e a divulgação do conhecimento em periódicos científicos ${ }^{(8)}$.

Resultado divergente foi encontrado em um estudo(7) que teve por objetivo analisar a tendência temática e metodológica das dissertações e teses do Programa de Pósgraduação em Enfermagem da Universidade Federal da Paraíba, ao constatar o predomínio dos estudos com abordagem qualitativa.

Corrobora com esse resultado, estudo com o objetivo de caracterizar as dissertações e as teses disponíveis no Catálogo de Teses e Dissertações (CEPEn) da Associação Brasileira de Enfermagem (ABEn), quanto ao caminho percorrido pela Enfermagem brasileira no âmbito do ensino, da pesquisa, da gestão e da prática assistencial. Os autores reiteram que a Enfermagem tem se preocupado em conhecer o ser humano em sua singularidade e complexidade, e a abordagem metodológica permite essa compreensão ${ }^{(9)}$.

Constatou-se no presente estudo, que o Estudo de caso, a Análise de conteúdo ou Temática e a Fenomenologia foram os referenciais teórico/metodológico predominantemente empregados pelos pesquisadores dos Programas mineiros de Pós-graduação.

Embora as Teorias de Enfermagem não estejam entre os referenciais mais adotados pelos pesquisadores, tem-se constatado a utilização crescente em dissertações e teses, o que corrobora com os achados do presente estudo ${ }^{(7)}$.

Há uma vasta possibilidade de referenciais metodológicos, e a opção de escolha pelo pesquisador, de acordo com o objeto de estudo, é parte do trabalho científico. Assim, essa escolha deve levar em consideração a coerência com o objetivo, com o problema, o campo a ser investigado e o referencial teórico, o que contribui para a qualidade da produção(10).

A opção metodológica pela análise de conteúdo e temática de Bardin e Minayo foram predominantes no presente estudo, assim como encontrado em outro estudo(11).

A análise de conteúdo consiste em um conjunto de técnicas de análise das comunicações visando obter, por procedimentos sistemáticos e objetivos de descrição do conteúdo das mensagens, indicadores, quantitativos ou não, que permitam a inferência de conhecimentos relativos às condições de produção/recepção dessas mensagens. A análise de conteúdo consiste na unidade de significação que surge naturalmente no texto analisado, a partir de uma afirmação a respeito de determinado assunto, ou seja, o revelar crítico da mensagem de maneira que se possa compreender sobre outra realidade que não aquela presente nela mesma ${ }^{(12)}$.

A fenomenologia permite uma estratégia qualitativa e interpretativa, visando ao conhecimento intersubjetivo, compreensivo e retratado, baseando na experiência de vida para compreender um fenômeno ${ }^{(13)}$. O uso desse referencial tem sido caracterizado pela grande diversidade de abrangência, refletindo-se em distintas abordagens e interpretações.

Martin Heidegger, um dos autores mais utilizados no estudo da fenomenologia, desenvolveu sua abordagem, por meio da fenomenologia hermenêutica, auxiliando na construção e fortalecimento dos pressupostos teóricos, contribuindo para as potencialidades científicas. Defende que a ciência moderna necessita do exercício da reflexão crítica, para a compreensão dos fenômenos humanos ${ }^{(13)}$.

$O$ estudo de caso é frequentemente situado dentro do paradigma qualitativo. É um método de pesquisa estruturado, e sua aplicação pode ser em diferentes circunstâncias, que contribui no campo de conhecimento tanto para os fenômenos individuais como grupais, sendo dotado de um conjunto de procedimentos lógicos e técnicas que permitem a operacionalização e relação entre causas e fenômenos ${ }^{(14)}$. O estudo de caso tem sido utilizado para responder questões complexas de saúde, e o pesquisador necessita de uma postura precisa e segura do método e atentar-se a todos os elementos do estudo de caso para promover a autenticidade, robustez e boa qualidade metodológica ${ }^{(15)}$.

Os estudos na área da enfermagem que utilizaram como referencial teórico-metodológico os pensamentos de Maffesoli têm aumentado, consideravelmente, trazendo importantes contribuições, desde a década de 90 até a 
atualidade. Esse referencial contribui para a atuação da equipe de enfermagem, mostrando diferentes caminhos na dimensão do cuidado, seja no campo assistencial ou científico, a partir do cotidiano das pessoas, buscando sua maneira de viver, ressignificando o cuidado que vai para além da técnica, possibilitando mudanças na maneira de olhar, para que possa entrar em sintonia com as diversas situações do viver e do conviver em sociedade, para um cuidado afetivo e efetivo ${ }^{(16)}$.

O referencial da Teoria das Representações Sociais possibilita o desenvolvimento de pesquisas com valorização da subjetividade das pessoas e também para a aplicabilidade no cuidado de enfermagem ${ }^{(17)}$. Propicia ainda, ao pesquisador a compreensão das atitudes, dos comportamentos e dos significados compartilhados por determinado grupo social, originando intervenções de enfermagem mais específicas e, consequentemente, mais eficientes, por respeitarem as características de cada grupo ${ }^{(17)}$.

A utilização desse referencial, pelos Programas de Pós-graduação mineiros, coaduna com os resultados de um estudo ${ }^{(7)}$ que, ao analisar o referencial teórico e metodológico de teses e dissertações, constatou que, de um total de 46 dissertações e teses analisadas, 32,6\% utilizaram a Teoria das Representações Sociais como arcabouço teórico, o que representou o segundo referencial mais adotado pelo Programa de Pós-graduação em Enfermagem da Universidade Federal da Paraíba. Observa-se, portanto, um alinhamento na opção teórica, o que aponta como importante perspectiva de parcerias para o desenvolvimento de pesquisas entre programas de regiões distintas.

A Hermenêutica-Dialética visa à compreensão e à crítica da realidade, por meio do entendimento de textos, dos fatos históricos e do cotidiano, permitindo identificar as diferenças, as controvérsias e a ruptura do sentido ${ }^{(4)}$. Utiliza a comunicação da vida cotidiana e do senso comum, fundamentando-se na experiência cultural, nas vivências, nos significados compartilhados e nos símbolos(4). Além disso, fornece os alicerces para a compreensão do sentido da comunicação, por meio da intersubjetividade, representando o movimento necessário à produção de racionalidade, em relação aos processos sociais complexos, ou seja, almeja apreender a prática social empírica dos indivíduos que compõem a sociedade ${ }^{(4)}$.
Constatou-se que o referencial da Hermenêutica-Dialética tem sido empregado nas dissertações e teses pela maioria dos programas, trazendo análises que possibilitaram compreender a intersubjetividade, vivências e os significados das pessoas dentro de uma sociedade complexa, imprevisível e mutável. Eles perceberam as contribuições desse referencial para a Enfermagem, uma vez que possibilita a identificação das contradições, convergências e divergências, atentando e orientando o enfermeiro em suas práxis ${ }^{(18)}$.

A Sociologia Compreensiva busca entender o inesperado, valoriza o casual, o banal, as apresentações incompletas da vida e as ações subjetivas das pessoas nos seus ambientes de relações. Remete à noção de espacialidade, em que o mundo é permeado por conflitos, ódio e amores, resultando em um lugar dinâmico, podendo ser chamado de socialidade. Ao oferecer diferente visão sobre as relações humanas, o presente referencial contribui às pesquisas científicas ${ }^{(19)}$, especialmente da área de Enfermagem, a qual é imprescindível compreender como cada pessoa vivencia o processo saúde-doença.

A Análise Compreensiva é um referencial que utiliza o procedimento analítico, requer que o investigador tenha a percepção enquanto sujeito compreensivo livre. Incentiva a interação de elementos e conexões em seu exercício, sendo como partes constituintes do universo multidimensional que integram o todo relativo ao sujeito $^{(20)}$. O pesquisador deve reproduzir a narrativa dos sujeitos, o conhecimento teórico e também expandir o seu próprio horizonte, com vista a interpretar e ser interpretado ${ }^{(20)}$.

O referencial de Análise Estrutural da narração é descrito por Demazière e Dubar ${ }^{(21)}$. Segundo os autores, a entrevista se constitui em uma oportunidade de reflexão para o entrevistado, o que resulta no vínculo com o entrevistador, de forma a possibilitar ao entrevistado apresentar sua análise dos fatos, justificativas e valores sobre eles e ao entrevistador avaliar, no decorrer das entrevistas, as categorias das representações ${ }^{(21)}$.

O método Etnográfico tem sido empregado nas dissertações/teses para a compreensão dos aspectos simbólicos da vida social e fundamentase na ideia de que os comportamentos humanos só podem ser compreendidos dentro do contexto sociocultural(22). Assim, a etnografia possibilita a 
dialogicidade entre o pesquisado e o pesquisador, permitindo, assim, uma troca mútua de saberes e experiências, o que torna importante para a Enfermagem compreender, na perspectiva da pessoa, seu modo de pensar e agir e suas experiências. Resultado similar foi encontrado em estudo na área da Enfermagem ${ }^{(23)}$ que aponta para a importância do uso desse referencial nas pesquisas científicas.

A Teoria Fundamentada nos Dados (TFD) objetiva compreender as experiências e as interações das pessoas inseridas em um determinado contexto social, buscando evidenciar as estratégias desenvolvidas diante dessas situações vivenciadas. A utilização da TDF em pesquisa na enfermagem mostra que o referencial está em consonância com a prática profissional do enfermeiro, pois esse assume papel crucial e fundamental na articulação das relações/interações no âmbito assistencial e organizacional e propicia uma intersecção entre paciente/família/profissionais/comunidade, com vista à busca de soluções e alternativas para os desafios e limitações que emergem na sua práxis ${ }^{(24)}$.

Em consonância com os achados do presente estudo, constatou-se que, de um total de 274 dissertações, a TFD predominou entre os referenciais metodológicos utilizados pelos discentes de Pós-graduação em Enfermagem da Universidade Estadual de Campinas, com um percentual de $35 \%{ }^{(8)}$, o que demonstra a relevância desse referencial para a pesquisa em enfermagem.

Em relação à produção internacional, há escassez de estudos que tenham por objetivo identificar os referenciais teórico e metodológico utilizados pelos Programas de Pós-graduação em Enfermagem. Estudo realizado entre as principais revistas do México com o objetivo de descrever as temáticas e características metodológicas das publicações de enfermagem, mostrou que dos 244 artigos publicados, 84\% não utilizaram teorias de enfermagem como referencial para orientar a pesquisa. Em relação aos estudos de abordagem qualitativa $(n=38)$, os referenciais mais utilizados foram Fenomenologia (35,55\%), TFD (19,3\%) e Etnografia $(6,4 \%)^{(25)}$.

Assim, a análise dos referenciais teórico/metodológico para a construção do conhecimento pela Pós-graduação mineira, aponta para a diversidade de opções que os pesquisadores se têm apropriado para a compreensão dos diferentes fenômenos e contextos relacionados ao processo saúde-doença e cuidado, contribuindo para a melhoria da práxis da enfermagem.

\section{CONCLUSÃO}

A investigação realizada teve a pretensão de oferecer ao leitor a visão de uma parte importante da construção do conhecimento dos Programas de Pós-graduação Stricto sensu em Enfermagem sobre os referenciais teórico/metodológico das dissertações e teses com abordagem qualitativa e mista.

A análise das dissertações e teses dos programas federais mineiros de Pós-graduação Stricto sensu em Enfermagem apresenta dados que apontam para o predomínio dos estudos com abordagem quantitativa e a incipiência em estudos mistos e Quali-Quanti.

Dentre os referenciais teórico/metodológico, constatou-se o predomínio da Análise de Conteúdo e Temática de Bardin e de Minayo, seguido pelo Estudo de caso e a Fenomenologia.

Neste trabalho, considera-se a importância da troca de experiências e parcerias entre os pesquisadores qualitativos dos cinco Programas de Pós-graduação Stricto sensu em Enfermagem, para a oferta de disciplinas e projetos de pesquisas compartilhados, de modo a ampliar as possibilidades na utilização de referenciais teórico/metodológico para a construção e divulgação do conhecimento, assim como, para o avanço da ciência da Enfermagem.

Desse modo, esses referenciais têm o potencial de alcançar o entendimento de como as pessoas compreendem seus universos cotidianos, propiciando ao pesquisador uma direção sobre como abordar os contextos individuais e sociais, de forma a contribuir para a translação do conhecimento e para a qualidade do cuidado de enfermagem.

A dificuldade no acesso a todas as dissertações e teses produzidas pelos Programas de Pós-graduação no recorte temporal estabelecido pode ser considerada uma limitação do estudo.

Constatou-se nos resumos de algumas dissertações e teses a incompletude dos dados relacionados aos referenciais teórico/metodológico, o que assinala para a necessidade de uma avaliação criteriosa da versão final do relatório de pesquisa. 
Sugere-se a realização de estudos entre outros Programas de Pós-graduação Stricto sensu em Enfermagem para conhecer uma parte importante da produção brasileira sobre os referenciais teórico/metodológico de estudos com abordagens qualitativa e mista.

\section{REFERÊNCIAS}

1- Mendonça GMMM, Cestari VRF, Rodrigues LN, Sampaio MOM, Freitas MC, Guedes MVC. Scientific production of grades of a postgraduation program in nursing. Rev Pesqui. 2018;10(2):485-89. DOI: $\quad$ 10.9789/21755361.2018.v10i2.485-489

2- Parada CMGL, Nichiata LI, Kantorski LP. A enfermagem no contexto da pós-graduação brasileira. J Nurs Health 2019;9(2):e199211. DOI: 10.15210/jonah.v9i2.16756

3- Bandeira AG, Witt RR, Lapao LV, Madruga JG. A utilização de um referencial metodológico na implementação de evidências como parte da investigação em enfermagem. Texto ContextoEnferm. 2017; 26(4):e2550017. DOI: $\underline{10.1590 / 0104-07072017002550017}$

4- Minayo MCS. O desafio do conhecimento: Pesquisa qualitativa em saúde. 13a ed. São Paulo: Hucitec; 2013.

5- Creswell WJ. Projeto de pesquisa: Métodos qualitativo, quantitativo e misto. 3a ed. Porto Alegre: Artmed; 2010.

6- Moreira SV. Análise documental como método e como técnica. In: Duarte J, Barros A, organizadores. Métodos e técnicas de pesquisa em comunicação. São Paulo: Atlas; 2005.

7- Pimenta CJL, Fernandes WAAB, Falcão RMM, Freitas AS, Oliveira JS, Costa KNFM. Análise das dissertações e teses do programa de pósgraduação em Enfermagem da Universidade Federal da Paraíba. Rev Min Enferm. 2018;22:e1093. DOI: $10.5935 / 1415-2762.20180023$

8- Reis JCC, Correia MDL, Botelho MTSL, Duran ECM. Knowledge production in a post-graduation program in nursing. Rev Enferm UFPE 2018;12(11):3052-9. DOI: 10.5205/1981-8963v12i11a236089p3052-3059-2018

9- Gomes ATL, Salvador P, Rodrigues CCFM, Assis YMS, Bezerril MS, Santos VE. Os caminhos percorridos pela Enfermagem brasileira na pesquisa: Estudo documental. Braz J Nurs. 2017;16(2):226. DOI: $\quad \underline{10.17665 / 1676-}$ $\underline{4285.20175451}$

10- Massaroli A, Martini JG, Lino MM, Spenassato D, Massaroli R. Método delphi como referencial metodológico para a pesquisa em enfermagem. Texto Contexto-Enferm. 2017;26(4):e1110017. DOI: $10.1590 / 0104-07072017001110017$

11- Gamarra TPN. Conceitos de saúde e doença: Análise das tendências em teses e dissertações brasileiras. Arq Ciênc Saúde UNIPAR 2019;23(1):49-55.

DOI:

\subsection{0/arqsaude.v23i1.2019.6588}

12- Bardin L. Análise de conteúdo. Lisboa: Edições 70; 2011.

13- Turcato JC, Sausen JFCL, Bartz CRF, Baggio DK. Contribuições da fenomenologia no domínio dos estudos organizacionais: Pesquisas de cunho interpretativo. Rev Univ Vale Rio Verde 2019;17(1):1-11. DOI: 10.5892/ruvrd.v17i1.5072

14- Andrade SR, Ruoff AB, Piccoli T, Schmitt MD, Ferreira A, Xavier ACA. O estudo de caso como método de pesquisa em enfermagem: Uma revisão integrativa. Texto Contexto-Enferm. 2017;26(4):e5360016.: DOI: 10.1590/0104$\underline{07072017005360016}$

15- Lentsck MH, Marcon SS, Baratieri T. Uso do estudo de caso qualitativo pela enfermagem brasileira: Uma revisão integrativa. Rev Enferm Atual In Derme. 2018;84(22):107-22. DOI: 10.31011/1519-339X.2018a18n84.9

16- Nitschke RG, Tholl AD, Potrich $T$, Silva KM, Michelin SR, Laureano DD. Contributions of michel maffesoli's thinking to research in Nursing and health. Texto Contexto-Enferm. 2017;26(4):e3230017. DOI: 10.1590/0104$\underline{07072017003230017}$

17- Pinheiro MGC, Rodrigues IDCV, Dias GA, Marcolino EC, Gomes BRS, Miranda FAN. Análise contextual da teoria das representações sociais na perspectiva da pesquisa qualitativa em enfermagem. Rev Enferm Cent-Oeste Min. 2019;8:e2722. DOI: 10.19175/recom.v8i0.3281

18- Corrêa VAF, Acioli S, TinocoTF. The care of nurses in the Family Health Strategy: Practices and theoretical foundation. Rev Bras Enferm. 
2018;71(Suppl 6):2767-74.DOI: 1 10.1590/00347167-2018-0383

19- Couto TM, Nitschke RG, Lopes RLM, Gomes NP, Diniz NMF. Cotidiano de mulheres com história de violência doméstica e aborto provocado. Texto Contexto-Enferm. 2015;24(1):263-9. DOI: $\quad$ 10.1590/0104$\underline{07072015003620012}$

20- Silva EFG, Santos SEB. Fenomenologia existencial como caminho para pesquisa. Rev NUFEN 2017;9(3): 110-26. DOI: 10.26823/RevistadoNUFEN.vol09.n03artigo17

21- Lages AS, França EB, Freitas MIF. Profissionais de saúde no processo de vacinação contra hepatite $B$ em duas unidades básicas de Belo Horizonte: Uma avaliação qualitativa. Rev Bras Epidemiol. 2013;16(2): 364-75. DOI: $\underline{10.1590 / S 1415-790 \times 2013000200012}$

22- Campos EA. Etnografia na pesquisa em saúde: Alcoolismo, doença e significado em um grupo de alcoólicos anônimos. Investigação Qualitativa em Ciências Sociais 2017 [citado em 14 fev 2020]; 3:537-45. Acesso em: https://proceedings.ciaiq.org/index.php/ciaiq2017 /article/view/1427

23- Ponce KLP, Tayabas LMT, González YC, Haro $\mathrm{OH}$, Zúñiga ML, Morán ACA. Nursing care for renal patients on hemodialysis: Challenges, dilemmas and satisfactions. Rev Esc Enferm USP 2019;53:e03502. DOI: 10.1590/S1980$\underline{220 \times 2018011103502}$

24- Klock P, Buscher A, Erdmann AL, Costa R, Santos SV. Best practices in neonatal nursing care management. Texto Contexto-Enferm. 2019;28:e20170157. DOI: 10.1590/1980-265xtce-2017-0157

25- Landeros-Olvera E, Ramírez-Girón N, YáñezLozano Á, Guzmán-Ramírez G, Galicia-Aguilar RM. Temáticas y características metodológicas de la investigación de enfermería publicada en revistas mexicanas: 2010-2015. Enfermería Universitaria 2018;15(3):274-83.

DOI:

\section{Editores Responsáveis:}

Juliana Dias Reis Pessalácia

Fabiana Bolela de Souza

Nota: Este trabalho contou com o apoio da Coordenação de Aperfeiçoamento de Pessoal de Nível Superior - Brasil (CAPES) - Código de Financiamento 001.

Recebido em: 09/09/2020

Aprovado em: $21 / 12 / 2020$

10.22201/eneo.23958421e.2018.3.65994 